\title{
Gradhiva
}

GRADHIV

Revue d'anthropologie et d'histoire des arts

$23 \mid 2016$

Collections mixtes

\section{Les collections d'art contemporain à l'épreuve du vivant à travers quelques cas remarquables}

Collections of contemporary art put to the test by the incorporation of living things: some remarkable case studies

\section{Cyrille Bret}

\section{OpenEdition}

\section{Journals}

Édition électronique

URL : http://journals.openedition.org/gradhiva/3170

DOI : $10.4000 /$ gradhiva.3170

ISSN : 1760-849X

\section{Éditeur}

Musée du quai Branly Jacques Chirac

Édition imprimée

Date de publication : 25 mai 2016

Pagination : 146-167

ISBN : 978-2-35744-093-7

ISSN : 0764-8928

\section{Référence électronique}

Cyrille Bret, «Les collections d'art contemporain à l'épreuve du vivant à travers quelques cas

remarquables », Gradhiva [En ligne], 23 | 2016, mis en ligne le 25 mai 2019, consulté le 01 mai 2019. URL : http://journals.openedition.org/gradhiva/3170 ; DOI : 10.4000/gradhiva.3170 

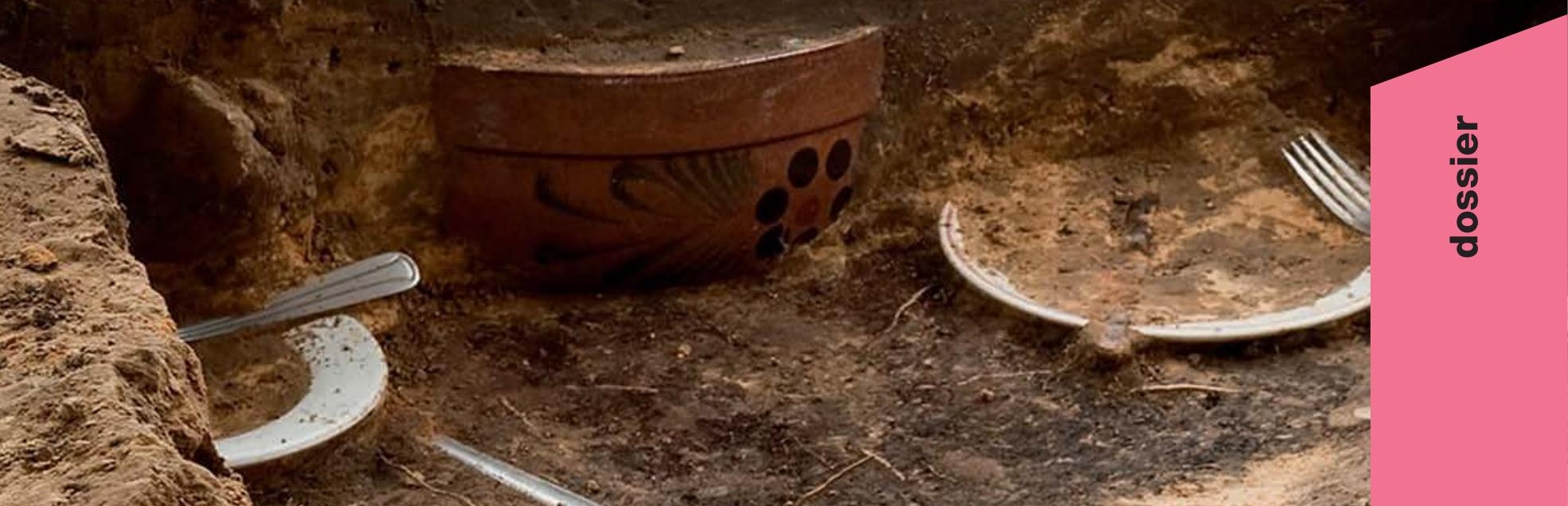

$x=$
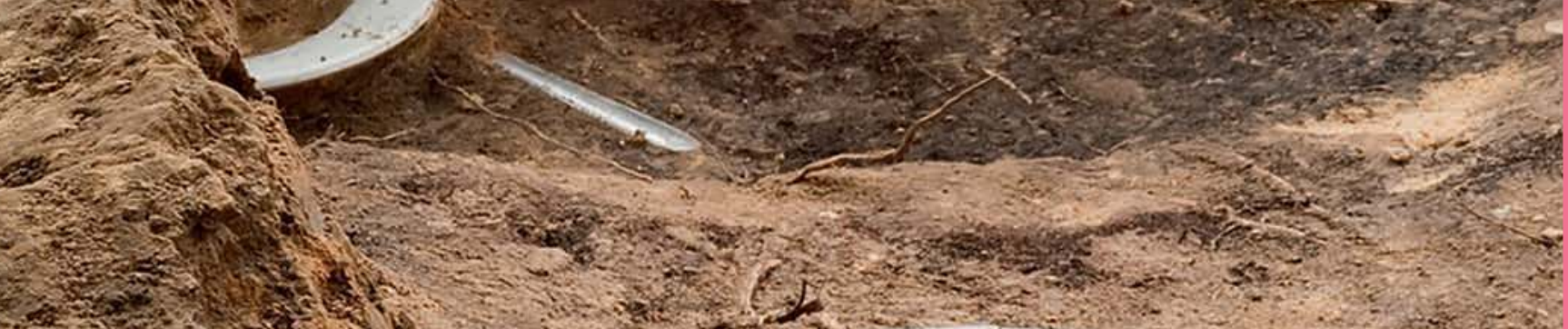

$\frac{1}{9}$
$\frac{0}{0}$
$\frac{0}{0}$

inting

675)

The

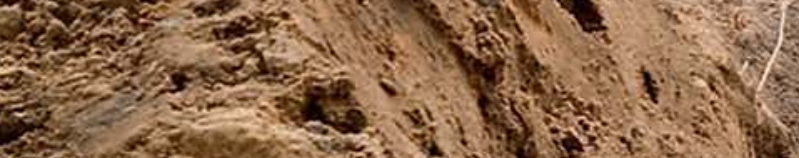
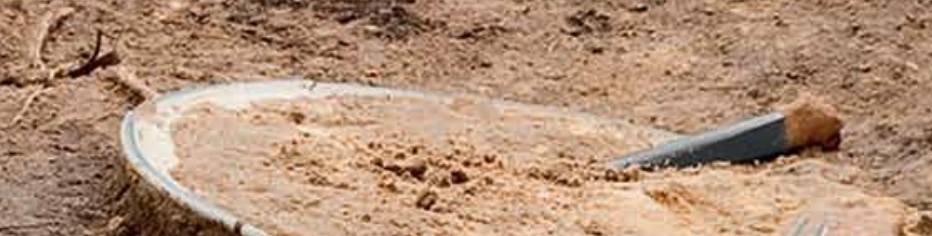

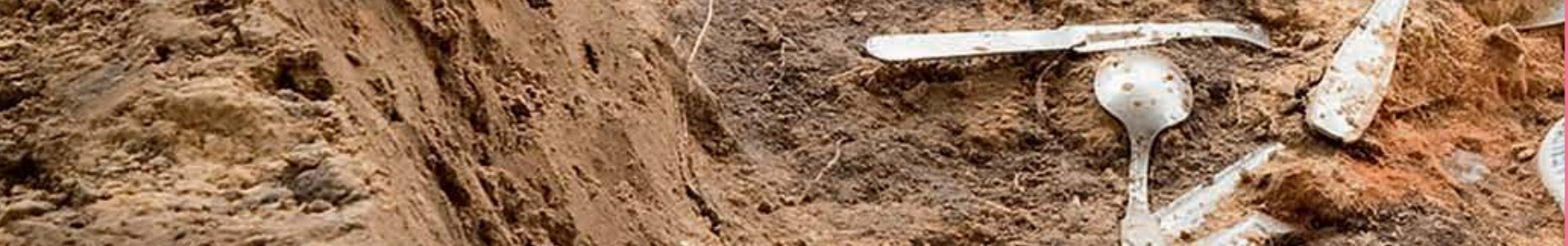

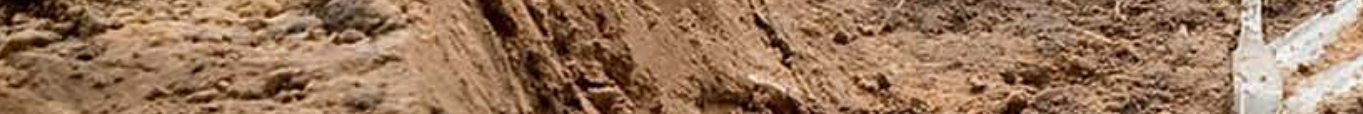

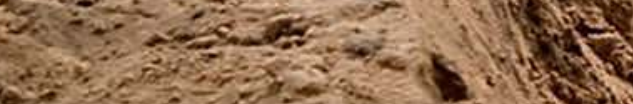

6)

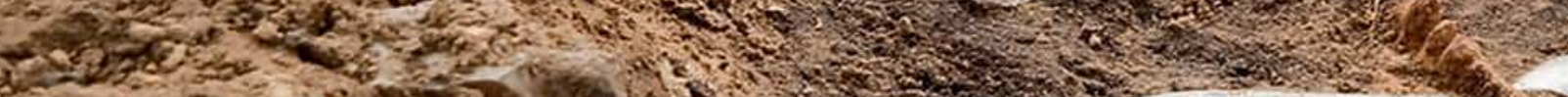

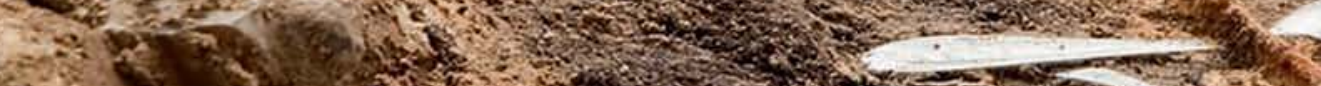

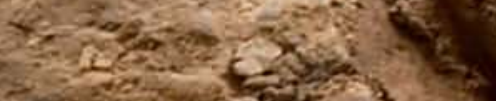

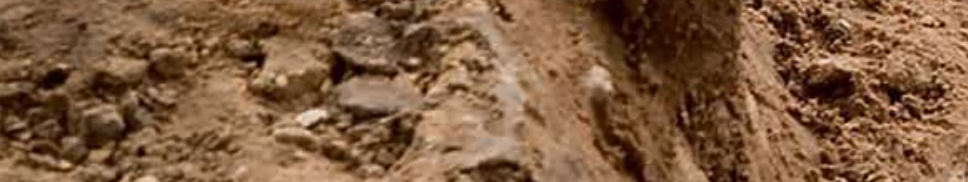

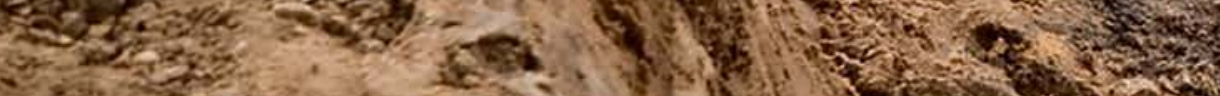

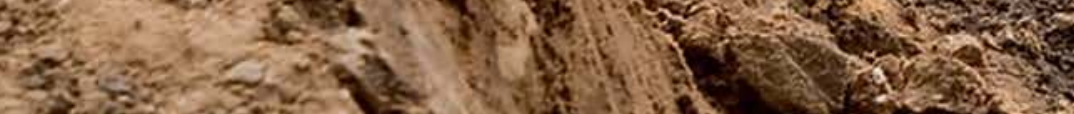

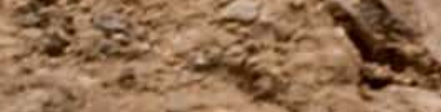

A. 1 is हx

Nit 3

ant

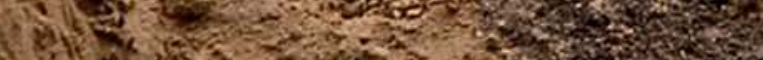

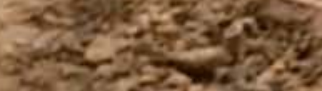

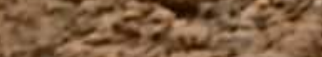

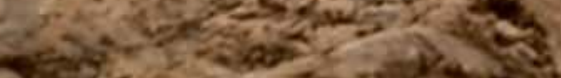

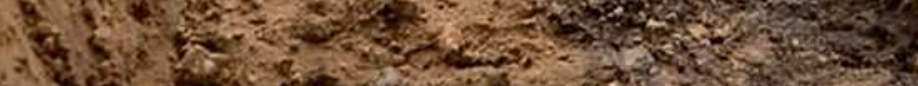

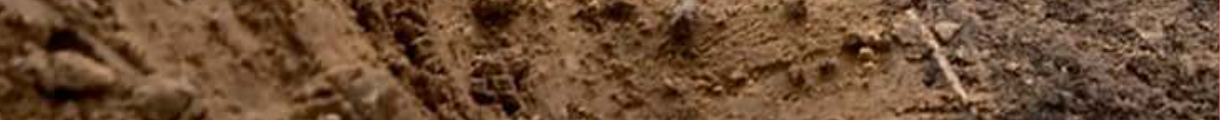

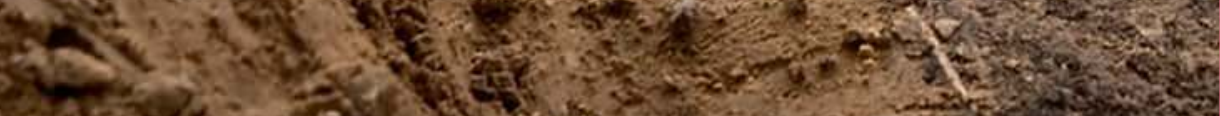
4 .

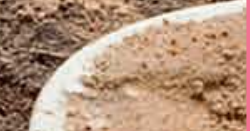




\section{Les collections d'art contemporain à l'épreuve du vivant à travers quelques cas remarquables}

par Cyrille Bret

L'œuvre d'art européocentrée s'est historiquement définie en tant qu'objet visuel unique. Or, à partir des années 1960, des artistes ont fait éclater ce modèle en artifiant le vivant et en révélant la portée artistique des clivages entre nature et culture, humain et non-humain. Dès lors qu'elles rentrent dans une collection, que deviennent ces œuvres mixtes mêlant le vivant à l'objet? À travers quatre études de cas récentes comprenant de la moisissure, des insectes, des animaux ou encore des corps humains, cet article vise à comprendre comment les chargés de conservation font exister ces entités composites dans une collection, et dans quelle mesure leur caractère vivant peut être préservé. Relevant les paradoxes de l'objectualisation de l'art et ses conséquences, il s'attarde sur le rôle de la durée d'exposition ou du protocole dans le bricolage des ontologies pluralistes et provisoires de ces œuvres. 


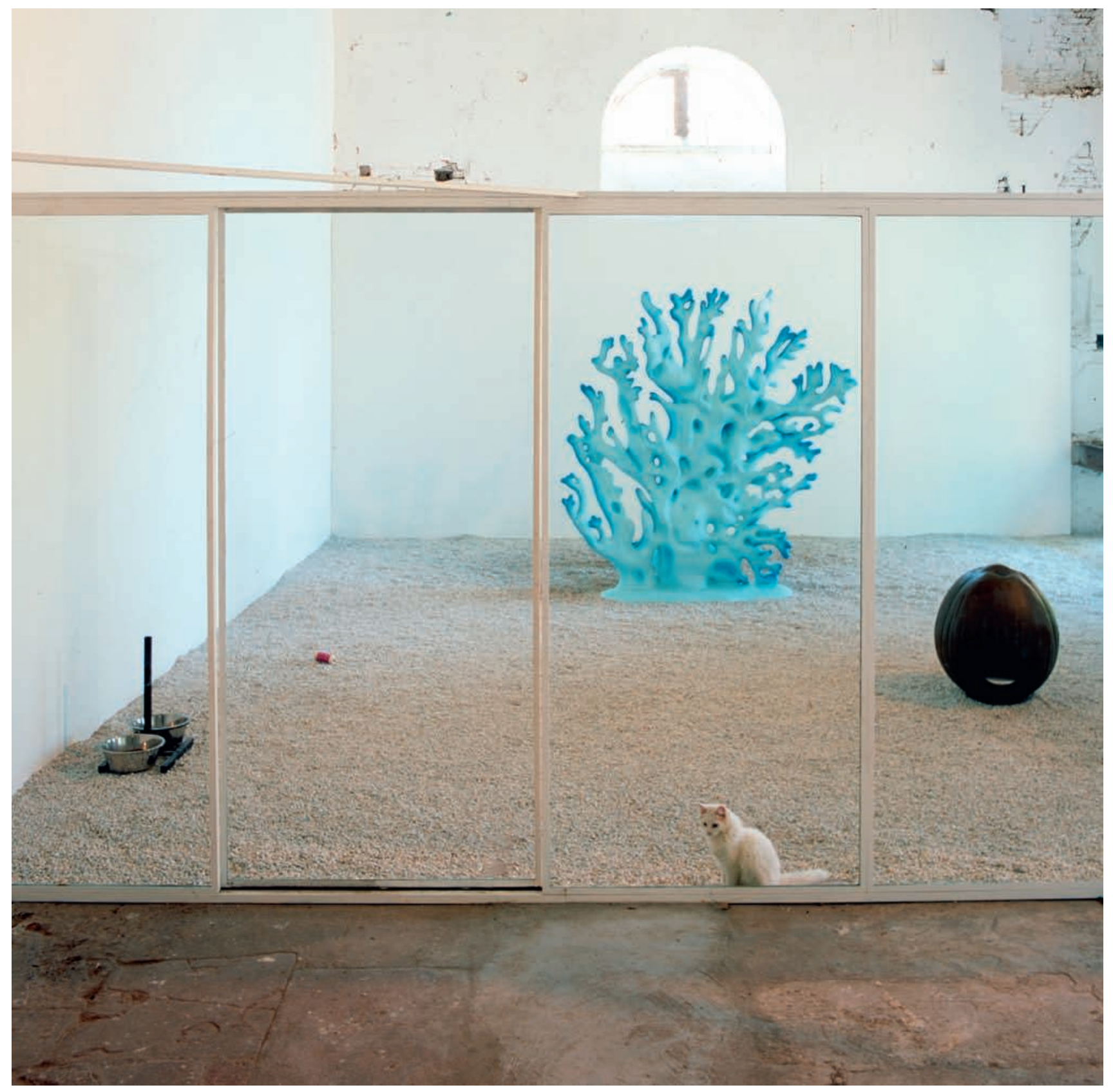


En 2007, alors que je travaillais pour l'Institut d'art contemporain de Villeurbanne, j'ai participé au montage d'une exposition monographique consacrée à l'artiste François Curlet dans laquelle une salle avait été intégralement réservée à la présentation d'une œuvre intitulée Chaquarium. Cette dernière avait alors parfaitement rempli cette fonction d'aiguillon spéculatif que l'on reconnaît parfois à certaines réalisations artistiques. Ainsi les visiteurs de l'exposition se retrouvaient-ils face à un simulacre d'aquarium d'environ $30 \mathrm{~m}^{2}$ (à l'échelle humaine donc). Une cloison en Plexiglas séparait l'espace des spectateurs, réduit à un couloir de passage, d'un monde métaphoriquement aquatique clos par des murs blancs sur les trois autres côtés, comprenant une sorte d'algue ou de corail monumental, du gravier blanc répandu au sol, une gamelle en inox et... un chat se prélassant dans une sculpture en forme de noix de coco creuse, à moins qu'il ne fût occupé à faire ses griffes contre le simili corail ou encore à se dandiner en faisant le tour du propriétaire. Cette installation n'a évidemment pas manqué de susciter de multiples discussions, qu'il s'agisse de visiteurs s'inquiétant du traitement réservé à l'animal durant l'exposition ou de certains collègues s'étant attachés au félin au point de vouloir l'adopter. Si l'iconologue ou l'interprète des images qu'est tout historien de l'art chercherait d'abord à saisir de quel énigmatique poisson ce chat est le prédateur et ce que son historicisation pourrait nous dire, d'autres questions pourraient in fine se révéler tout aussi capitales pour une histoire de l'art soucieuse de saisir l'ensemble des dynamiques et des interactions sociales tramant ce que par consensus on appelle œuvre d'art: qu'advient-il du chat lorsque l'exposition se termine? Peut-il quitter à si bon compte l'assignation ontologique et juridique que lui confère sa présence sur la notice décrivant matériellement l'œuvre (qui le place entre la résine polyester et l'inox)? Comment décrire le type de collectif social auquel il participe? Qu'adviendrait-il enfin du chat si l'installation était patrimonialisée?

À travers quatre études de cas en situation de collection comprenant respectivement un organisme simple auto-extensif, une dizaine d'insectes sculpteurs, cent cinquante pigeons ou encore un certain nombre de corps humains en action, cet article vise à comprendre les moyens pratiques dont disposent les personnes chargées de leur conservation ${ }^{\mathbf{1}}$ pour faire exister des entités mixtes (objet/vivant) dans une collection, et dans quelle mesure il leur est possible de conserver peu ou prou vivante ${ }^{2}$ la partie reconnue comme telle du dispositif dont ils sont responsables. Pour ce faire, je vais m'autoriser à comparer des situations patrimoniales contemporaines proches les unes des autres, mais diverses si on les envisage du strict point de vue de leurs typologies esthético-matérielles (installations, sculptures ou performances), en m'attachant moins aux œuvres elles-mêmes qu'aux effets que leur agencement situationnel produit. Gageons que le chat persan peuplant de sa majestueuse présence le Chaquarium de Curlet puisse nous servir d'intercesseur tout au long des pages qui suivent.

\section{Quelques remarques historiographiques et méthodologiques} sur le clash de (l'histoire de) l'art et du vivant

II me serait difficile de débuter mon analyse sans préciser qu'envisager le dépassement et/ou l'intrication (ce sont là deux options distinctes) entre nature et culture du point de vue de l'histoire de l'art n'est possible
1. Cet article n'entend pas circonscrire la collection et la conservation au seul champ muséal, ce qui serait éminemment réducteur compte tenu du fait que toutes les collections d'art contemporain ne sont pas liées à des musées (rien qu'en France, il faut aussi compter les Fonds régionaux d'art contemporain, le Centre national des arts plastiques, les centres d'art dotés d'une collection), ni ne relèvent du seul domaine public (voir les fondations et collections privées d'art contemporain). Bien évidemment, ces différences de cadre sociojuridique s'accompagnent de variations notables de statut et de mission des personnes chargées de conserver les œuvres. On connaît les conservateurs et attachés de conservation travaillant dans les musées publics ou assimilés, mais il existe aussi des chargés de collection, de conservation, ou encore des régisseurs qui exercent une mission de conservation dans de nombreux autres contextes institutionnels.

\section{ci-contre}

fig. 1

François Curlet,

Chaquarium, 2003.

Vue de l'exposition

Chaquarium, Cimaise et Portique, Albi, 2003; verre (paroi), peinture acrylique sur résine polyester (corail), métal et plastique (ventilateur, machine à bulles), résine polyester (caillou), système électrique, gravier, Cococat (résine polyester. coussin), chat persan, inox et acier (écuelles et portant acier), litière pour chat; corail, $210 \times 210 x$ $40 \mathrm{~cm}$; circa $30 \mathrm{~m}^{2}$.

Avec l'aimable autorisation de l'artiste et Air de Paris, Paris (C) Photo Marc Boyer. ADAGP, Paris 2016.
Bien que ces individus aient en commun le souci technique de conserver, c'est-à-dire a minima de maintenir en l'état les œuvres dont ils ont la charge, tous n'ont pas à exercer par exemple de mission scientifique, de mise en valeur, d'enrichissement ou encore d'étude et de classement à l'endroit de ce qu'ils conservent, et donc d'obligation à se conformer strictement à des règles et à une déontologie de la conservation soumise au contrôle du service des Musées de France. Cet article s'intéresse donc moins aux règles de conservation qu'aux enjeux et aux conséquences des pratiques de conservation au sens large dans le champ pluriel des collections d'art contemporain.

2. Dans la suite de l'article, le terme "vivant » est employé au sens commun de l'autonomie active dont sont dotées certaines entités qualifiées d'organismes ou d'êtres, sans rentrer dans le détail des caractérisations plus approfondies relevant des sciences du vivant (médecine, biologie...). Ce parti pris tient au fait que les acteurs manipulant ce concept dans le champ de l'art contemporain utilisent principalement ce terme en ce sens. 
3. À l'appui de ces quatre paradigmes, je ne peux, faute de place, que renvoyer tout d'abord à l'émergence de l'iconologie comme science des images et des formes de pensée visuelle, ainsi qu'en témoigne la place principale qu'elle occupe dans la méthodologie de l'histoire de l'art, mais aussi pour les autres points aux travaux de Michael Baxandall sur l'étude des contrats au Quattrocento (Baxandall 1985), à ceux d'Édouard Pommier sur l'invention de "l'art » à la Renaissance (Pommier 2007), ou encore aux descriptions économiques de l'œuvre, comme chez Raymonde Moulin (Moulin 2003). Bien entendu, il existe un grand nombre d'autres travaux dans la littérature grise que l'on pourrait mobiliser à bon droit pour garantir chacun de ces quatre paradigmes. Je ne peux cependant m'empêcher de souligner ce passage de Pierre Francastel qui évoque (non sans déférence envers la sociologie durkheimienne) «les objets esthétiques des civilisations" (Francastel 2006: 39).
4. Je concentrerai mon propos sur le living art, que l'histoire de l'art de cette période a peu analysé sous cet angle, quand bien même cela m'oblige à laisser de côté certaines œuvres de l'Arte Povera comme Alpi Marittime. Continuerà a crescere tranne che in quel punto, de Giuseppe Penone, de 1968. Pour cette pièce, l'artiste a réalisé un moulage en acier de sa main enserrant le tronc de l'arbre, lequel croît ensuite en intégrant cet élément étranger au prix d'une déformation à l'endroit de cet assemblage. On trouvera maints exemples de ce genre chez Yannis Kounellis Mario Merz ou encore Giovanni Anselmo, pour lesquels les analyses ne manquent pas.

5. Ce «multilivre» (comme le désigne l'artiste) regorge de prises de position comme celle-ci : "L'art ne doit pas se développer dans le vide, en se référant exclusivement à l'art. De toute façon, nous ignorons ce qu'est l'art. La seule référence doit être la vie et vivre. " (Filliou 1998: 93)

6. Le terme "multiple" est apparu dans le même contexte pour désigner une pratique de démultiplication de l'œuvre d'art (selon une logique proche de l'édition en petites séries) visant à contourner le règne de l'unicum et le fétichisme de l'original qui lui est attaché (avec le multiple, on ne peut plus identifier l'original de ses copies). qu'à condition de ré-historiciser le concept d'œuvre d'art, pris au sens d'une construction sociale européocentrée qui s'est sédimentée depuis la fin du Moyen Âge européen autour de quatre paradigmes dominant la production d'artefacts culturels (sans pour autant être exclusifs): 1) la primauté accordée au visuel sur les autres sens; 2) l'objectualité comme condition sociale principale, pour ne pas dire déterminante; 3) l'affirmation d'une unicité sémiomatérielle (corrélative à la condition d'objet); 4) le règne de l'unicum comme principe économique de structuration du champ de l'art ${ }^{3}$. Cet espace logique de l'œuvre d'art produit par les conflits ou les continuités entre les différents mondes de l'art qui ont traversé l'histoire européenne entre la fin du Moyen Âge et le $x \mathrm{XII}^{\mathrm{e}}$ siècle (sans compter d'autres facteurs antérieurs d'origine principalement gréco-romaine), les acteurs européens du champ artistique (artistes, commanditaires, commentateurs) l'ont affiné par leurs interactions sociales jusqu'à ce qu'il fasse l'objet d'un consensus relativement stable. Ce processus s'est par ailleurs trouvé considérablement renforcé par l'essor de la réflexion sur les pratiques patrimoniales depuis la fin du XvIII siècle, jusqu'à inspirer les catégories juridiques du droit patrimonial. Au cours du $x x^{e}$ siècle, les éléments de la nature semblent avoir été exclus du périmètre de ce qui définit, de façon quasi générique, l'œuvre d'art, sinon à l'état mort ou figuré, ce qui procède assez logiquement du grand partage entre nature et culture et du regard naturaliste qui le fonde, décrit par Philippe Descola comme opérant une continuité de la matière et une discontinuité des esprits (Descola 2005: 176) associée à une domination du sujet transcendantal humain sur le monde.

Si l'on quitte l'échelle de la réflexion historiographique et la longue durée propre à saisir les agencements sociaux pluriséculaires dans leurs dynamiques évolutives pour s'attacher aux événements advenus sur les différentes scènes de l'art depuis les années 1960, en Europe et en Amérique du Nord, force est de constater que les débats qui ont animé certaines collectivités artistiques n'ont cessé de mettre en jeu une forme d'incompatibilité renforcée (une tension contraire) entre nature et culture. Ainsi, dans les années 1960 , les mots living en anglais et «vivant» en français ont fait leur apparition dans les débats artistiques pour qualifier l'émergence du champ des pratiques performatives dans le sillage des premiers happenings sur les scènes nord-américaine et européenne ${ }^{4}$. Jusque dans le milieu des années 1970, avant que le terme de performance se généralise, celui-ci est encore souvent concurrencé par l'appellation «art vivant/living art», puis définitivement préempté par le milieu du théâtre où le «spectacle vivant » finit par désigner les enjeux d'un théâtre élargi. Sur la scène new-yorkaise, entre 1959 et 1970, on voit la troupe de théâtre expérimental du Living Theatre de Julian Beck et Judith Malina participer à la nébuleuse des happenings initiée notamment par les artistes Allan Kaprow et Al Hansen. Quelques années plus tard, Robert Filliou, lui-même un temps associé à cette scène et dont l'engagement pour lier poésie, art et action n'est plus à démontrer, n'hésite pas à titrer l'un de ses livres les plus célèbres Teaching and Learning as Performing Arts $^{\mathbf{5}}$ (Filliou 1998). Prolongeant ce type de réflexion dans un autre registre, l'artiste Fluxus Alison Knowles, elle aussi associée aux premiers events de 1964 dans l'espace public à New York, avait développé un an auparavant une série de multiples ${ }^{6}$ intitulée Bean Rolls. Chaque exemplaire de Bean Rolls se compose d'une boîte en étain comportant une 
étiquette offset et contenant des haricots secs, métaphorisant une logique d'autopoïèse. Pour ce type de haricot, la germination est dite inhibée jusqu'à ce que le contact avec l'humidité et certaines conditions de température et de luminosité enclenchent la reprise du processus métabolique (au maximum 72 heures après humidification la germination devient épigée). L'éventualité de la germination est cependant limitée à cinq ans. Celle-ci et l'artification des haricots permettent l'élaboration d'une représentation du vivant produisant des êtres sériels mais non identiques par laquelle l'artiste manipule, sur un plan métaphorique, les catégories conceptuelles de l'unique et du multiple: une dichotomie qu'elle entend ici dépasser. Enfin, comment ne pas évoquer cet autre exemple célèbre, lorsqu'en 1974 Joseph Beuys s'enferma une semaine durant avec un coyote pour un exercice de diplomatie territoriale performée intitulé I like America and America likes me, cherchant à panser symboliquement, dans les termes de son vocabulaire plastique, les plaies du grand schisme entre nature et culture. Ces différentes tentatives ont connu des destinées variables en termes de patrimonialisation. Les performances n'ont véritablement trouvé leur place dans les collections des musées qu'avec la production de vidéos (les premières en format U-matic), accompagnant le questionnement esthétique autour du statut des œuvres et des documents, tandis que le coyote beuysien n'accéda au musée que sous la forme d'un « récit autorisé7 " (Poinsot 1999: 135) associé à quelques rares clichés photographiques. Seuls les Bean Rolls ont eu l'honneur de passer la porte d'une réserve sans faire l'objet d'une métamorphose lorsque les époux Gilbert et Lila Silverman, collectionneurs historiques de Fluxus, décidèrent de léguer leur collection au Museum of Modern Art (MoMA) de New York en 2008. II faut dire qu'en cas de germination, le haricot fautif est promptement enlevé.

Le terme «vivant » n'en est donc venu à décrire que plus tardivement une dimension pour ainsi dire matérielle des œuvres d'art. Le point commun entre ses différentes acceptions (et ce qu'elles recoupent) en usage dans l'histoire des idées artistiques des cinquante dernières années en Europe et en Amérique du Nord est de contribuer à décrire la façon dont une œuvre parvient à déjouer les clôtures de l'objet et acquérir, moyennant quelque artification ou bricolage ontologique, une forme d'autonomie processuelle couplée à une énergie intrinsèque. Parmi les multiples cas existants, il n'y a parfois rien de plus qu'une continuité métaphorique avec le topos esthétique de l'anima ${ }^{8}$. N'oublions pas cependant que, pour les artistes des années 1960, et particulièrement les Américains, la recherche d'une dimension processuelle est directement corrélée à la construction d'un regard nouvellement informé par des philosophies extrême-orientales (bouddhisme zen, taoïsme...) particulièrement prisées dans les milieux artistiques de l'époque (Bret 2014). Se rejoue ici une propension à renouveler les arts grâce à une attention aux conceptions extra-occidentales de la nature (phénomène assez analogue, indépendamment du contexte, à celui de l'apparition de l'Art nouveau à la fin du XIX ${ }^{\mathrm{e}}$ siècle $^{9}$ ). Cette vision du vivant n'a donc que peu de rapport avec une vision biologiste qui, dans les années 1960, mettait davantage l'accent sur les paramètres de l'évolution et de l'individuation, à la lumière des recherches sur la vie cellulaire et de la découverte de l'ADN. Pour achever cette mise au point, il convient également de rappeler une évolution récente des musées d'art contemporain: à partir du milieu des années 1980, l'entrée d'une œuvre
7. L'historien de l'art JeanMarc Poinsot a élaboré ce concept pour saisir la multiplicité des récits secondaires participant de l'œuvre et qui ne relèvent ni de la performativité linguistique propre à tout ou partie de celle-ci (partition, statement conceptuel...), ni à des énoncés théoriques reconnaissables comme tels, mais à des récits «institués» qui accompagnent l'œuvre un peu comme des légendes (cartels, certificats, notices, déclarations d'intention, interviews, catalogues, témoignages, documents, etc.). Ces récits sont au cœur d'un " contrat iconographique " par lequel se manifeste une "figure de l'artiste", soit la manière dont ce dernier "exerce ses prérogatives d'auteur et le champ qu'il leur donne". (Poinsot 1999: 136) Dans le cas du coyote, celui-ci se retrouve comme "ventriloqué " par la documentation de la performance.

8. L'anima est un terme latin (lié morphologiquement et métonymiquement à "animal ") aux multiples usages et acceptions, que Descartes, dans son Discours de la méthode, envisage comme un souffle primordial qui informe la matière inerte pour en faire un corps vivant. Dans les conceptions artistiques européennes à l'âge classique (en continuité avec des idéaux esthétiques gréco-romains), ce terme se rencontre parfois pour désigner ce qui paraît vif dans une figuration, voire pour métaphoriser une impulsion vitale.

9. Il s'agit donc là d'une attention naturaliste au sens artistique du terme, et non plus au sens anthropologique développé par Philippe Descola. 
fig. 2

"Mode d'emploi »/livret

d'accompagnement

de l'œuvre, conçu et

dessiné par l'artiste.

Coll. les Abattoirs-Frac

Midi-Pyrénées, Toulouse.

Courtesy de l'artiste et Art:

Concept, Paris (C) Adagp,

Paris, 2016.

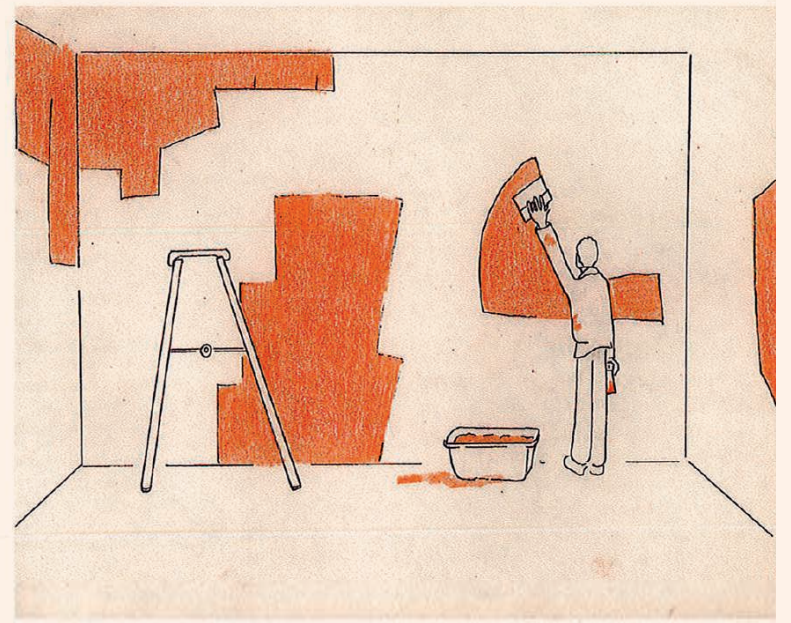

Enduisez par zones discontinues.

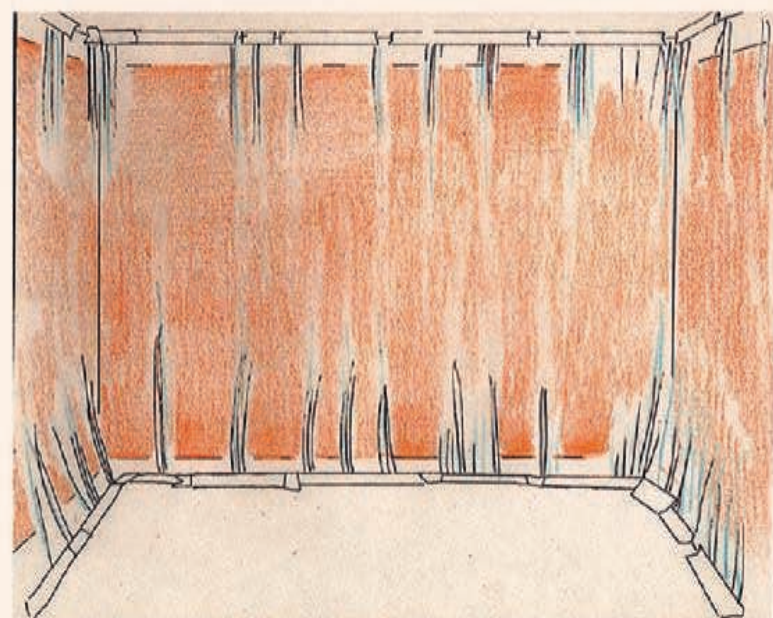

Tendez une bâche à $15 \mathrm{~cm}$ de la surface enduite. La bâche doit être placée dès que les murs sont enduits.

Surveillez le développement des moisissures. Attendez 8 jours et enlevez la båche.

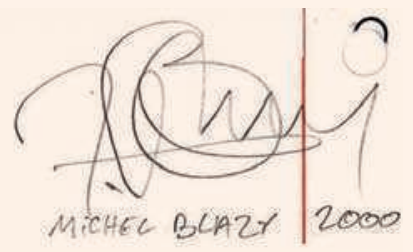

\section{Les murs de poils de carotte}

Vous pouvez enduire un seul mur, deux, trois, ou la totalité des murs de la salle d'exposition.

En séchant la matière se rétracte, se fissure et tombe en croûtes, ces résidus doivent rester au sol.

\section{réalisation}

Une personne capable d'enduire un mur avec du plâtre.

Les murs doivent rester enduits et la bâche posée en un seul jour. Si la surface à enduire est trop importante, faites appel à des assistants.

\section{matériaux}

- purée de carottes congelée

- purée de pommes de terre en flocons

- bâche plastique fine de protection

- scotch large transparent

\section{outils}

- spatule large

- échelle

- spatule mince

- grands bacs plastique

- cuillère longue 
10. Dans les années 1980 , Thierry Raspail et Thierry Prat, respectivement conservateur et régisseur, ont été chargés de fonder une collection et un musée d'art contemporain à Lyon, et se sont notamment inspirés du modèle du New Museum pour expliciter l'esprit de leur projet scientifique et culturel. Cette manière de construire une collection avait l'immense intérêt de correspondre parfaitement aux pratiques de délégation de production, grandissantes depuis les années 1970, et typiques du conceptualisme et des formes de l'installation.

Le rôle de régisseur s'en est trouvé accru au point que ce genre d'établissement est devenu le lieu d'un équilibre inédit entre conservateur et régisseur.
11. Dans son étude des pratiques de conservation au MoMA, Fernando Dominguez Rubio s'est attaché à décrire le caractère docile ou unruly («indiscipliné») d'œuvres ne relevant pas à proprement parler de la dichotomie nature/ culture, mais révélant comment le musée, qu'il qualifie de «machine à objectualiser », affecte les propriétés des œuvres en les transformant en objets montrables, classifiables et connaissables.

12. L'œuvre est depuis conservée dans la collection des Abattoirs-FRAC MidiPyrénées (Inv. 2001.2.22). L'ensemble des informations qui suivent est consultable sur le site des Abattoirs: http://www.lesabattoirs. org/blog/des-histoiresdoeuvres/michel-blazymur-de-poils-de-carotte2000 (consulté le 8 novembre 2015).

13. Vidéomuseum est un dispositif numérique qui vise à centraliser et mettre en réseau des informations muséographiques concernant les œuvres d'art contemporain dans les collections publiques françaises. C'est aussi un important vecteur d'uniformisation des pratiques muséographiques, malgré de profondes différences institutionnelles. dans des collections publiques se fait de moins en moins par la voie de l'achat ou du don mais plutôt par celle de la production, suivant le modèle muséologique développé spécifiquement pour l'art contemporain au New Museum, à New York, par Marcia Tucker, et qui a suscité un vif intérêt y compris dans les musées européens ${ }^{\mathbf{1 0}}$. J'arrêterai là cet état des lieux visant à mettre en perspective les études de cas qui vont suivre. Ne pouvant traiter l'ensemble des possibilités ouvertes par la question du vivant dans les collections d'art contemporain, y compris en me limitant à la période allant de 1960 à nos jours, je me suis attaché à mettre en lumière quatre configurations ou situations actuelles relevant d'un rapport entre l'art, le vivant et une collection, et ce afin d'illustrer, autant que faire se peut, les assertions qui suivent:

A. L'intégration d'éléments vivants au sens commun du terme dans tout ou partie des constituants sémio-matériels de l'œuvre tend à mettre à l'épreuve l'ontologie des quatre paradigmes dominants de l'œuvre d'art énoncés plus haut, et engager ainsi un rapport polémique avec cette histoire et l'institution qui la porte, à savoir le musée, sa fonction de patrimonialisation et ses collections, celui-ci fonctionnant comme objectification machine ${ }^{\mathbf{1 1}}$ (Dominguez Rubio 2014 : 617-645).

B. Néanmoins, les acteurs sociaux (humains et non humains) du monde de l'art ne cessent par leurs pratiques de coopérer pour surmonter ce régime de contradiction.

\section{Quatre études de cas prises dans l'histoire (du temps présent) de l'art contemporain} Un organisme simple auto-extensif dans une collection publique française

L'artiste Michel Blazy (né en 1966) s'est fait une spécialité des installations ou des dispositifs implémentant des formes de vie micro-organiques, comme dans le Mur de poils de carotte, réalisé en 2000 pour les Abattoirs de Toulouse ${ }^{\mathbf{1 2}}$. La consultation du protocole de l'œuvre rédigé par l'artiste nous indique que sa réalisation nécessite de la «purée de carottes congelée», de la "purée de pommes de terre en flocons» (deux composants classés dans la catégorie «matériaux») et "une personne capable d'enduire un mur avec du plâtre », autant que possible en «un seul jour », à quoi s'ajoutent des "outils» comme des bâches de plastique transparent, du scotch, une échelle, une spatule, etc. S'ensuivent divers conseils concernant les quantités de purée à utiliser en fonction des dimensions des murs à recouvrir, ou encore le temps de décongélation nécessaire. La suite est accompagnée de croquis assez précis, dûment légendés, documentant les différentes étapes de la réalisation ainsi que les gestes techniques à accomplir (notamment l'application d'une bâche à distance du mur enduit afin de contrôler l'avancée de la moisissure dans les premiers jours suivant l'installation). Enfin, le texte comprend des indications relatives au rendu de l'œuvre: «En séchant la matière se rétracte, se fissure et tombe en croûtes, ces résidus doivent rester au sol. » La notice Vidéomuseum ${ }^{\mathbf{1 3}}$ la décrit comme suit: «CEuvre en trois dimensions, installation murale: murs d'une pièce recouverts d'un enduit organique. Mode d'emploi, ingrédients: purée de carottes, purée de 
pommes de terre, eau, CD-Rom documentaire. " Comme dans le cas du Chaquarium évoqué en introduction, cette liste permet de fixer des entités dont l'agencement fait œuvre et, là encore, la juxtaposition énumérative qui prévaut en matière de muséographie semble ramener sur le même plan des éléments aux qualités fort distinctes. En somme, retenons qu'en pratique l'œuvre est reconstruite pour chaque nouvelle exposition, qu'elle s'apparente à une sorte de culture de mousses dont la charge émotionnelle tient à son fort impact chromatique, à sa dimension immersive englobant le spectateur et à l'image de murs en état de décrépitude, comme si l'assise du white cube $\mathbf{1 4}^{\mathbf{1 4}}$ était métaphoriquement attaquée par des micro-organismes. L'idée qui préside à l'émergence de ce mode de monstration et qui a conduit au cours du siècle dernier à imposer un cadre d'exposition normatif (murs blancs, vide spatial autour des œuvres, éclairage zénithal...) procède de la croyance en l'élimination de tout ce qui relève de la sphère sociale - le mythe formaliste de l'autonomisation du regard, parallèle au mythe moderniste de l'autonomie de l'art. On ne peut qu'être frappé par la similitude entre les raisons qui ont motivé le white cube et celles qui ont présidé à l'élimination du facteur social dans les protocoles d'expérimentation scientifique en laboratoire, si bien décrits par Bruno Latour ${ }^{15}$. Compte tenu des normes de conservation en vigueur dans les collections publiques, le chargé de collection se doit de recréer les conditions de maintien de l'œuvre en l'état et empêcher sa dégradation en modulant si besoin les paramètres d'humidité, de température ou de luminosité (ce qui peut s'avérer difficile dans certains lieux). II doit aussi tout faire pour que les mousses ne se répandent pas en dehors du périmètre qui leur est assigné, qu'elles ne contaminent aucune paroi adjacente et que les murs qui supportent la moisissure en soient le moins abîmés possible. Une fois l'exposition passée, il ne conserve que le protocole - le vivant naturalisé (pasteurisé?) sous forme de préparation industrielle étant racheté pour chaque nouvelle exposition - tandis que la régie et l'équipe de démontage s'emploient à «remettre au propre» l'espace, ce qui suppose, dans pareil cas, le recours à des produits phytosanitaires de type fongicide (parfois inclus dans certaines peintures). L'artiste lui-même, comme en témoigne son protocole, a intégré ces contraintes dès le processus de création, ce qui nous fait dire que le régime du regard naturaliste prévaut en tout point dans cette installation relevant davantage d'une bio-ingénierie esthétique permettant une expérience de pensée qui articule le vivant et le régime objectuel de l'art que d'une abolition de la dichotomie nature/culture. En d'autres termes, l'artiste a culturalisé le micro-organisme selon un procédé propre au naturalisme qualifié par Descola de «relativisme absolu» (Descola 2005: 417-419), qui prend la nature pour un réservoir de formes et de symboles dans lequel l'humain vient puiser.

\section{Une dizaine d'insectes sculpteurs}

\section{dans une collection publique française}

Hubert Duprat (né en 1957) construit son rapport au vivant en déléguant quelque chose du pouvoir de l'artiste à des insectes, comme dans l'installation intitulée Trichoptères, datant de 1997. II travaille depuis les années 1980 avec des larves de phryganes (famille des trichoptères, comprenant environ douze mille espèces d'insectes) qu'il collecte dans des cours d'eau. À l'état larvaire, ces proches cousines des papillons ont la
14. Sur le white cube, je renvoie à l'analyse désormais classique de Brian O'Doherty (O'Doherty 2008).

15. Sauf erreur de ma part, cette histoire comparée reste largement à écrire. 


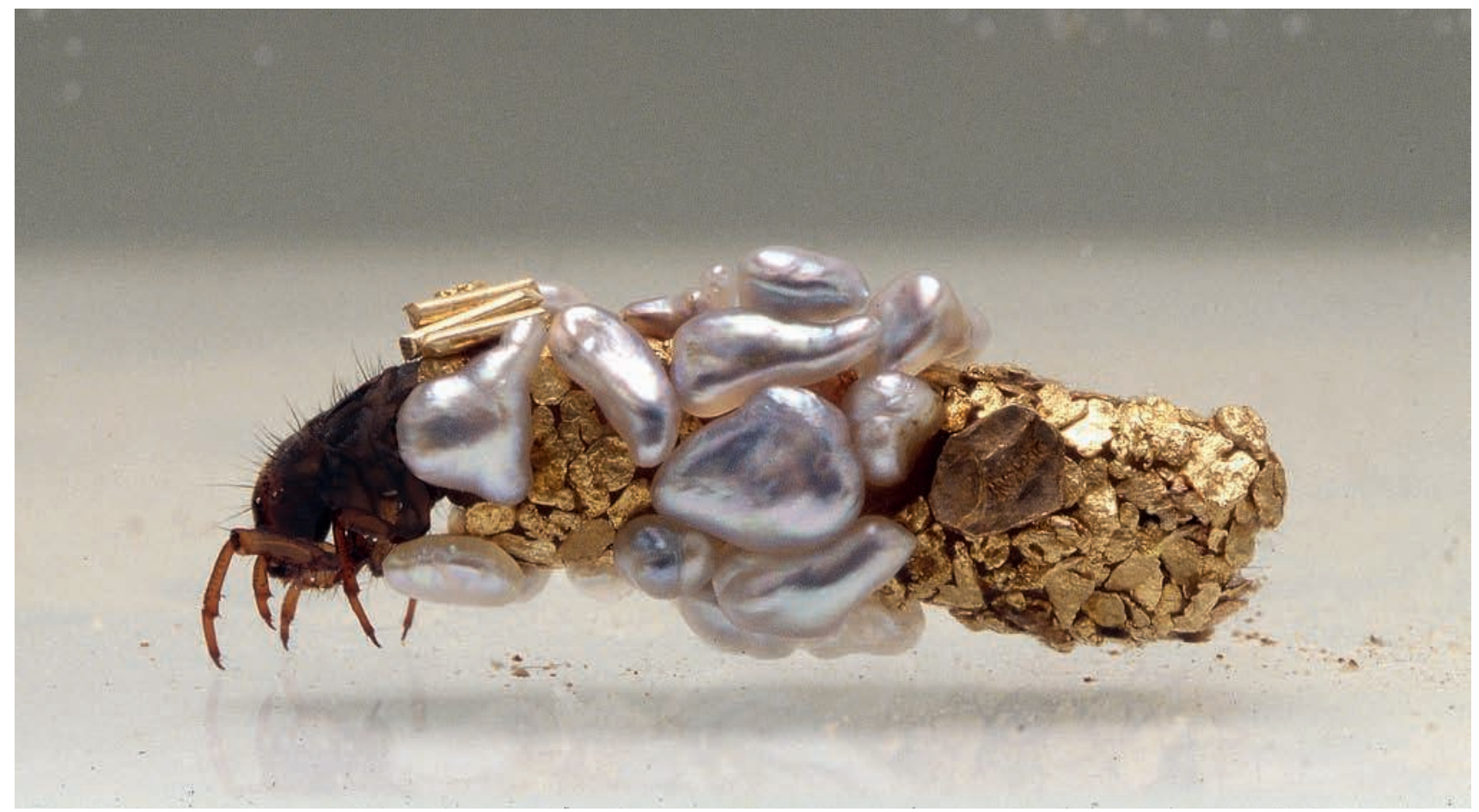

fig. 4

Hubert Duprat, Larve de trichoptère avec son étui (vue d'exposition), 19802000, or, perles. Photo: Frédéric Delpech / avec l'aimable autorisation de l'artiste et Art: Concept, Paris. ADAGP, Paris, 2016.

fig. 5

Hubert Duprat, Tube de trichoptère, 1980-2012, paillettes et bâtonnets d'or, perles, turquoise, 0,9x

$1,9 \mathrm{~cm}$. Collection privée, Paris. Photo: Fabrice

Gousset / avec l'aimable autorisation de l'artiste et Art: Concept, Paris. ADAGP, Paris, 2016.

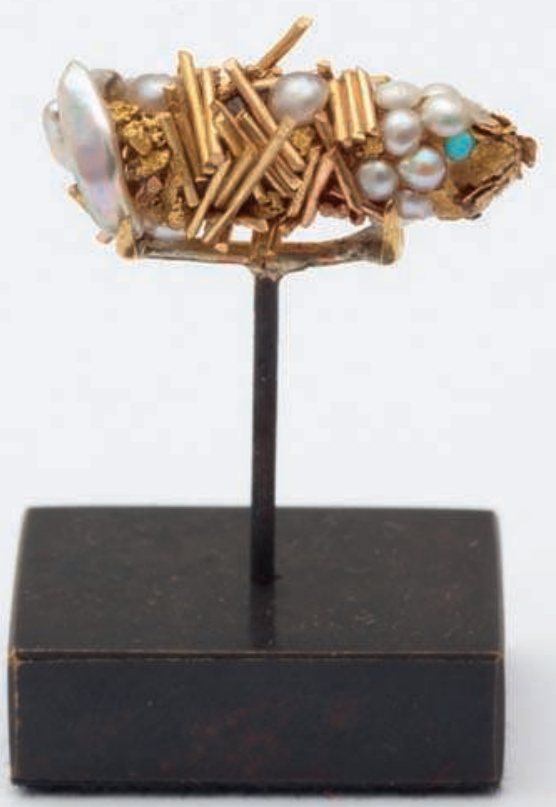


particularité de se forger un cocon de protection au moyen de petits graviers ou de brindilles glanés dans leur environnement, processus qui peut durer plusieurs mois avant qu'elles opèrent leur transformation. Duprat capture des phryganes qu'il élève ensuite dans des aquariums, en leur donnant d'autres matériaux que ceux de leur environnement naturel: des pierres, des métaux précieux, de la nacre, etc., faisant ainsi apparaître ces insectes comme des orfèvres, voire plus simplement comme des sculpteurs. L'installation dont il est question ici a été réalisée pour la collection du château d'Oiron, institution qui a la particularité d'être un monument historique combinant une relecture du dispositif des anciens cabinets de curiosités à une collection provenant du Fonds national d'art contemporain. Samuel Quenault, qui occupe la double fonction de régisseur et de chargé de collection, a publié un article très détaillé relatant par le menu les multiples actions qu'il a dû réaliser durant dix ans, à raison d'un à deux mois par an, pour maintenir cette œuvre en état d'équilibre et garantir la mixité vivant/objet. En voici quelques passages particulièrement éclairants sur son travail durant les deux mois de l'exposition:

Cela demandait donc des manipulations, et des soins dispensés aux animaux. [...] Pendant ces deux mois de présentation/production, mon travail consistait tous les matins à les sortir des bacs à eau réfrigérée à $5^{\circ} \mathrm{C}$, à les placer dans cet aquarium un peu incliné, et tous les soirs à les replacer chacun dans des bacs différents (contenant les matériaux précieux), et évidemment à les nourrir. J'allais acheter du poisson à la poissonnerie, et, je ne rentrerai pas dans les détails administratifs, mais c'est très compliqué quand vous travaillez pour une administration de vous faire rembourser des reçus de poissonnerie ou ce genre de chose. (Quenault 2013)

La suite de son récit mentionne les problèmes de mortalité des trichoptères, leur tendance au cannibalisme en cas de déficit de nourriture, sans compter les complications liées à l'extraction de leur fourreau ou le risque de les retrouver coincés dans des parties fonctionnelles de l'œuvre. L'un des passages les plus intéressants concerne les questions d'assurance. Chaque étui étant assuré (du fait de son statut patrimonial et des frais de restauration qu'un sinistre occasionnerait), Quenault évoque la difficulté pour un assureur de prendre en compte l'éventualité d'une destruction de son fourreau par l'insecte lui-même. Aucune clause dans le droit des assurances ne permet en effet de couvrir correctement les risques encourus par de tels dispositifs. II signale ainsi l'intervention d'un restaurateur qui venait suppléer à la fragilité des substances sécrétées par l'insecte en les consolidant avec du Paraloïd $®$, une résine acrylique utilisée dans le champ de la restauration des œuvres d'art. Le chargé de conservation devait alors s'essayer à "convaincre [sic] l'insecte à sortir doucement de l'étui sans qu'il casse celui-ci» (ibid.). Cet exemple témoigne du bricolage ontologique, juridique et patrimonial qu'un chargé de collection a dû inventer et exercer quotidiennement, faisant de cette pièce un exemple relativement rare de coopération entre humains et non-humains à l'intérieur d'une situation patrimoniale. 
16. L'Assemblée nationale française a voté le 28 janvier 2015 une modification du Code civil (art. 515-14) reconnaissant que l'animal est "un être doué de sensibilité ". Cet amendement de la pensée naturaliste ne va cependant pas jusqu'à entériner la reconnaissance d'une intériorité subjective aux animaux, et ne concerne pas les insectes (comme les phryganes) ou les micro-organismes (comme la moisissure de purée de carottes)..

\section{ci-contre}

fig. 6

Kader Attia, Flying Rats, 2008, installation éphémère, Lyon,

La Sucrière (Biennale d'art contemporain); cage grillagée contenant 150 pigeons vivants et 45 mannequins d'enfants habillés mais dont le corps est constitué d'un mélange de mousse et de graines alimentaires. ADAGP,

Paris, 2016. Photo Laurent Lecat.
Cent cinquante pigeons dans une collection privée

Lorsque l'on passe à la catégorie des animaux, d'autres enjeux semblent se dessiner, par exemple une possibilité accrue d'intersubjectivité entre humains et non-humains. Lors de la Biennale d'art contemporain de Lyon en 2005, l'artiste Kader Attia (né en 1970) a réalisé une installation, coproduite par la galerie Kamel Mennour, qui figure une cour d'école entourée d'une cage grillagée haute de plusieurs mètres dans laquelle des sculptures anthropomorphes (des enfants) constituées de grains agglomérés, portant vêtements et perruques, sont soigneusement dévorées par une centaine de pigeons. Laissons de côté la dimension spectaculaire doublement surdéterminée par une animalité largement dépréciée dans l'inconscient collectif (la pièce s'intitule comme de juste Flying Rats) et par le souvenir latent des Oiseaux d'Alfred Hitchcock, pour nous intéresser aux tribulations économiques de l'œuvre. À l'issue de la biennale, l'installation, dont la description matériologique sur le cartel indiquait «Cage, sculptures en graines pour oiseaux, perruques, sacs, 150 pigeons. Dimensions variables », et dont le coût de production avait été de 50000 euros, est vendue 60000 euros au célèbre collectionneur suisse Pierre Huber, qui la cède à son tour à l'occasion de la vente aux enchères de sa collection chez Christie's (New York) en 2011 pour un prix réalisé de 90000 dollars. Nonobstant la plus-value substantielle qu'il ne faudrait pas attribuer trop vite au nouveau statut ontologique des pigeons (il ne s'agit pour eux que d'un statut intermédiaire), mais bien davantage à l'intelligence marchande de Huber, notons que, dans le cadre de collections privées, c'est la logique du contrat qui prévaut, ellemême subordonnée à un cadre juridique de référence (ce qui vaut aussi pour les collections publiques) qui dépend du contexte territorial dans lequel l'installation est située. Or toutes les législations nationales ne sont pas équivalentes s'agissant du statut et des caractéristiques juridiques accordés (ou non) aux animaux. Faut-il rappeler qu'au moment où l'œuvre est produite à Lyon, le droit français ne voyait alors en l'animal qu'un «bien meuble ${ }^{\mathbf{1 6}}$ »? Les obligations vétérinaires (nombre de mètres carrés par volatile, type de vaccination, nourriture et suivi vétérinaire, conditions de repos des volatiles et de gestion de leur stress) varient donc également, comme le contrat et les obligations respectives de l'éleveur et de l'utilisateur (artiste, galeriste, collectionneur). Cette œuvre, en revanche, ne vit littéralement pas en dehors des périodes d'exposition (à la différence des phryganes de Duprat), un collectionneur (une fondation ou une institution publique) préférant louer les pigeons plutôt que d'avoir à se transformer en éleveur aviaire... Durant les périodes d'exposition, la personne chargée de la conservation de l'œuvre se doit de veiller aux flux de public, à l'éclairage et au niveau d'exposition sonore (installer une œuvre au niveau sonore élevé dans une salle voisine est à éviter) car ces volatiles sont sujets au stress (qui peut être un facteur de mortalité). II faut également être attentif aux échelles de température, gérer la collecte des déjections (dont l'accumulation serait susceptible de heurter trop violemment l'odorat des visiteurs) ou encore désactiver l'alarme du lieu lorsque celle-ci fonctionne par détection de mouvements. Bref, il s'agit de mettre en place tout ce qui est requis par la législation et le vétérinaire pour que les pigeons ne tombent pas malades (l'œuvre doit-elle être désinstallée en cas d'épidémie de grippe aviaire?), quand il ne convient pas tout bonnement de freiner la boulimie des volatiles menaçant de faire trop rapidement disparaître les sculptures anthropomorphes. 


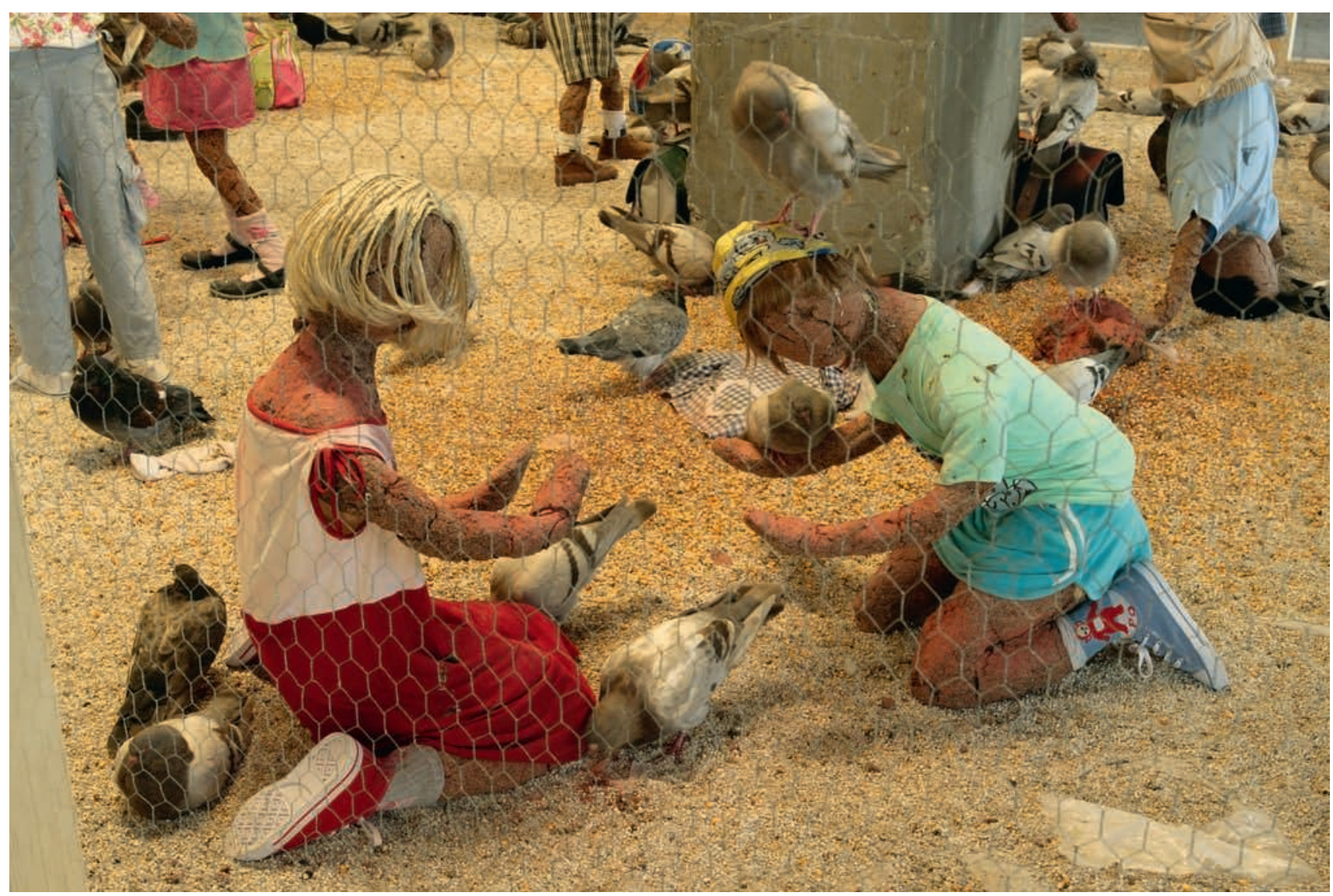


17. Ce texte, qui tient lieu d'avertissement au public et reprend les codes d'un document muséographique de type cartel allongé (aux informations habituelles s'ajoute un commentaire permettant de comprendre le fonctionnement de l'œuvre), a été produit par le musée d'Art contemporain de Montréal et contrevient assez explicitement au contrat qu'il évoque; [en ligne], disponible sur: http://www. macm.org/expositions/ tino-sehgal (consulté le 8 novembre 2015).

18. Descola cherche à dépasser l'opposition entre "relativisme absolu " et «monisme naturaliste» en proposant le concept de «relativisme relatif» qu'il emprunte à Bruno Latour. Ce concept "[...] ne part pas de la nature et des cultures, des substances et des esprits, [...] mais des relations de continuité et de discontinuité, d'identité et de différence, de ressemblance et de dissimilitude que les humains établissent partout entre les existants " (Descola 2005 : 419).

\section{Des corps humains en action}

dans une collection publique au Québec

Pour achever ce tour d'horizon de l'actualité du vivant dans l'art contemporain, essayons d'amender le regard naturaliste qui est de facto celui de I'historien de l'art, et de sortir le corps du performeur des schèmes discursifs auxquels il est réduit. L'artiste Tino Sehgal (né en 1976) a décidé d'investir le champ de la performance en créant, en 2007, une pièce intitulée This Situation acquise par le musée d'Art contemporain de Montréal. Des acteurs ont alors été recrutés localement dans les milieux universitaire et chorégraphique (cela a son importance pour Sehgal, qui vient de l'univers de la danse) pour engager la discussion avec le public de manière accidentelle ou fortuite, dans l'espace d'exposition, autour de questions telles que l'esthétique de l'existence ou le passage d'une société de la pénurie à une société de l'abondance. Voici comment le musée décrit ces œuvres résolument non objectuelles - mais surtout pas immatérielles -, le contrat qui encadre leur vente, ainsi que leur existence juridique, patrimoniale et esthétique:

En considération du rejet absolu, par Sehgal, des objets manufacturés, le processus d'acquisition d'une de ses œuvres consiste en une transaction purement orale engageant l'artiste ou l'un de ses représentants, la direction, la conservation et le registrariat du musée, et un juriste. Les conditions d'acquisition et d'installation de l'œuvre sont énoncées, et ainsi mémorisées par tous les assistants; le prix est négocié et, quand les deux parties parviennent à un accord, on se serre la main. Aucun document écrit n'accompagne cette démarche. Les conditions de présentation spécifient la rémunération de tous les interprètes en plus d'une stricte interdiction de captation vidéo ou photographique, d'impression de communiqués de presse, d'un catalogue, de cartels ou de panneaux didactiques ${ }^{\mathbf{1 7}}$.

Cet exemple pourrait de prime abord apparaître comme étant hors de propos ou comme un cas limite dans le cadre de cette argumentation. Pourtant, il est tout à fait remarquable que le texte ne mentionne que de manière indirecte, presque in absentia, ce qui conditionne l'existence même du projet, à savoir des humains bien vivants (des corps) sans lesquels This Situation ne pourrait tout simplement pas avoir lieu. Ainsi, l'utilisation prédominante de la voie passive renvoie le lecteur à un rite d'interaction schématisé et abstrait plutôt qu'aux actions pratiques qui lui donnent vie. Impossible de patrimonialiser une interaction (seule l'épreuve performative permet d'évaluer celle-ci), encore moins les individus qui l'activent; en revanche, un musée peut acquérir un protocole. Cet oubli permanent de l'action des corps humains au profit d'une substantialisation de leur intentionnalité ramenée à des structures discursives, des scripts d'interaction, voilà quelque chose que Descola qualifierait peut-être de «monisme naturaliste», soit un naturalisme entendu comme l'unique principe de rapport au monde, cimentant définitivement le partage entre nature et culture jusqu'à en oublier certaines qualités relevant du registre (dominé) des physicalités, pendant du « relativisme absolu » dont il était question à propos de l'installation de Blazy ${ }^{18}$ (Descola 2005: 417-419). Si ce réductionnisme protocolaire est d'autant plus problématique, concernant le travail de Sehgal, que ce dernier pense ses performances comme des chorégraphies dans lesquelles le rapport entre les corps des acteurs 
et des spectateurs, les déplacements et les gestes (la parole a souvent valeur de geste verbal) sont des éléments essentiels de son vocabulaire formel, il témoigne néanmoins d'une forme de médiation ontologique permise par ce dispositif 19 .

\section{Conclusion: Pour une histoire (de l'art) du grand partage}

Que nous enseignent ces différents cas sur les moyens pratiques dont les chargés de collection disposent pour faire exister des entités mixtes (objet/vivant) dans des collections patrimoniales ou privées tout en conservant peu ou prou vivante la partie qualifiée comme telle du dispositif?

Premièrement, pour les œuvres d'art contemporain mixtes (objet/ vivant) relevant de gestes de production, de construction ou d'assemblage, plutôt que de gestes de collecte - à l'exception des stratégies post-readymade -, la grammaire naturaliste si bien décrite par Descola (Descola 2005) prime toujours, malgré les velléités réelles des artistes de secouer l'esprit de l'art et ses institutions sociales, de formuler artistiquement des agencements sociocognitifs relevant d'une forme d'expérimentation sociale. Deuxièmement, il s'agit donc en dernier ressort, pour l'élément qualifié de vivant, d'exister dans un référentiel objectuel, visuel, forclos et unique, qui forme l'espace logique de cette réflexion et le mode d'existence dominant pour l'œuvre d'art. Par conséquent, les chargés de collection, les artistes et la plupart des acteurs de chaque monde de l'art concerné n'ont pas d'autre choix que de mettre en place des pratiques de collaboration avec d'autres acteurs, fussent-ils non humains, à travers des collectifs sociaux ou des médiations qu'il sera sans doute plus facile de décrire en partant des théories de Latour (Latour 2006: 93-101), particulièrement lorsqu'il propose "d'élargir la gamme des acteurs", que de celles d'Howard S. Becker autour des phénomènes de chaînes de coopération strictement anthropocentriques (Becker 1988). Albena Yaneva (Yaneva 2003) fournit un bon exemple de ce type d'analyse dans son étude sur une exposition au musée d'Art moderne de la Ville de Paris en 1999, qui privilégie le point de vue des coulisses du travail de l'art et de ses multiples acteurs sur celui du visiteur, et dont l'approche s'inspire de la tradition ethno-méthodologique (analyse conversationnelle et théorisations sociales des acteurs) et d'une sociologie latourienne propice à étudier «l'art en action», sur le modèle de la «science en action». Cette approche, que d'aucuns pourraient juger trop tournée vers les sciences sociales, gagne à être croisée aux théories esthétiques actuelles, et plus particulièrement à celle de Jean-Pierre Cometti qui déconstruit la séparation des sphères esthétique et sociale ${ }^{20}$, refusant de dissocier les modes de production, d'activation ou encore de reconnaissance des œuvres d'art de leur ontologie, censément éphémère, plurielle, voire «friable» (Cometti 2012).

Parmi les pratiques identifiées dans nos quatre études de cas, deux ressortent en particulier. Tout d'abord, la stratégie visant à résorber la durée de vie de l'élément «de nature» pendant l'exposition. En effet, le temps d'exposition, par ses bornes, permet d'objectualiser l'expansion propre au vivant. Pourrait-on dire de ce procédé qu'il relève d'une sorte d'artification du processus d'émergence ${ }^{21}$ ? Sans doute, mais pour l'affirmer avec robustesse, encore faudrait-il travailler sur un corpus plus conséquent,
19. Pour un élargissement et un approfondissement des enjeux relatifs

à la patrimonialisation des pratiques performatives, on consultera avec profit l'ouvrage récemment édité par Teresa Calonje (Calonje [éd.] 2014).

20. À la suite des philosophes pragmatistes (John Dewey notamment) et néo-pragmatistes auxquels il a consacré de nombreuses études.

21. J'entends ici par processus d'émergence une idée autant discutée par les philosophes que par les physiciens, les chimistes et les biologistes depuis le xix siècle. En biologie, le bon fonctionnement de chacun des constituants d'un système biologique ne garantit pas à l'ensemble du système d'être vivant. Le concept d'émergence permet de dépasser l'opposition entre vitalisme et matérialisme tout en démontrant que la complexité peut surgir de formes simples sans pour autant s'y réduire de manière mécaniste.

\section{double page} suivante

fig. 7

Michel Blazy, vue de l'exposition Monanism, an evolving exhibition, MONA, Hobart (Tasmanie), 2011. Avec l'aimable autorisation de l'artiste et Art: Concept, Paris. ADAGP, Paris, 2016. Photo: Rémi Chauvin. 


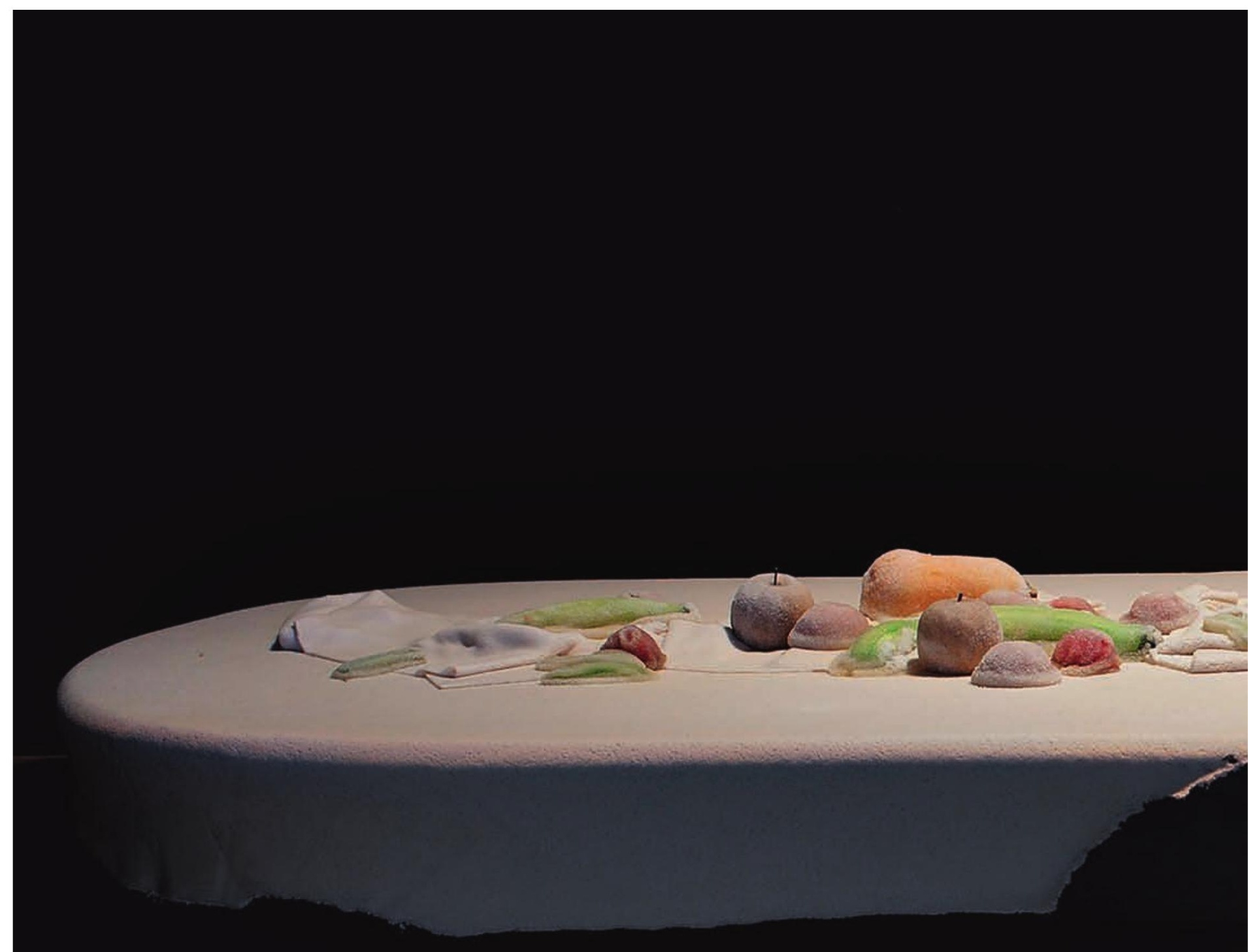



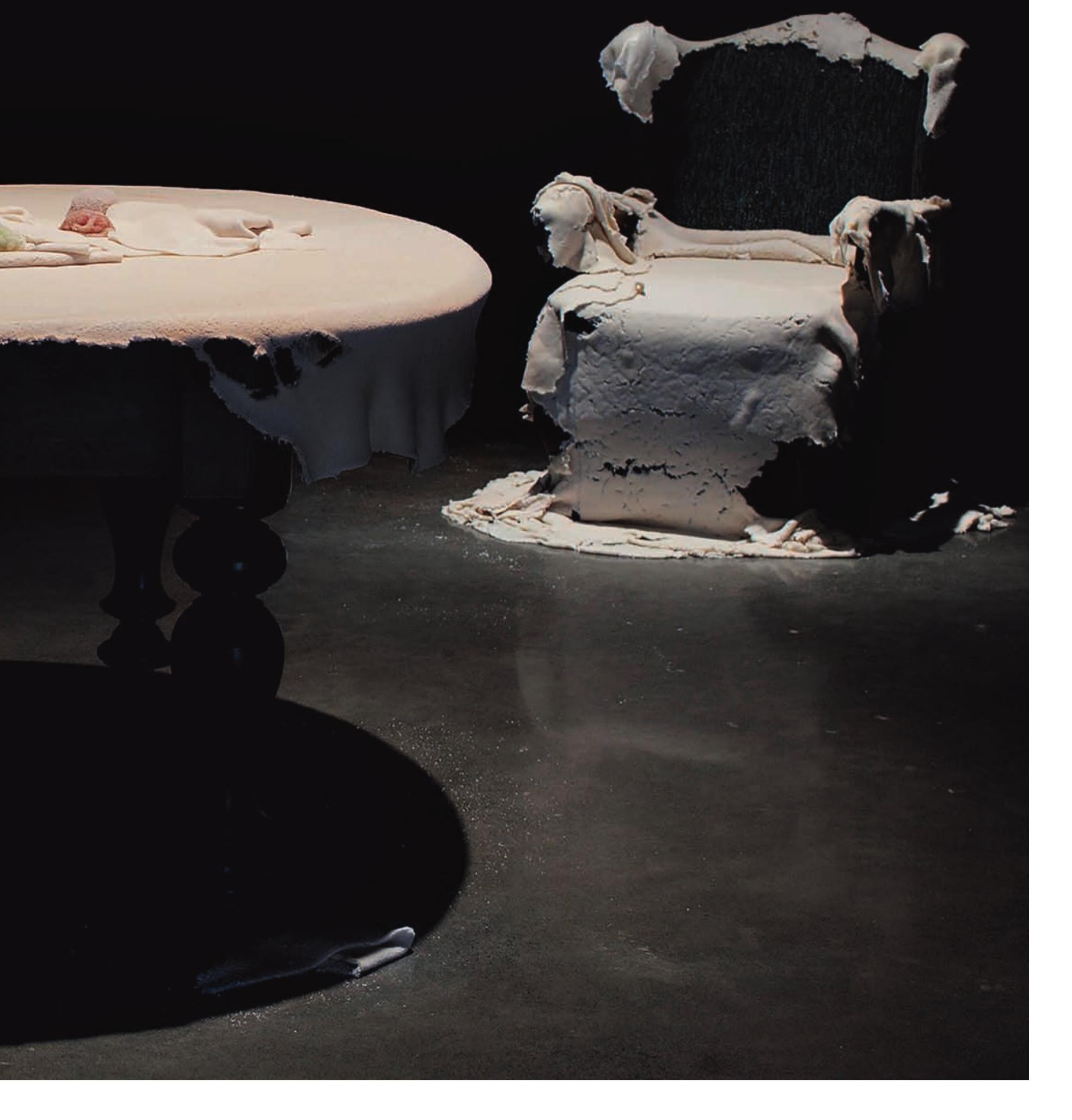


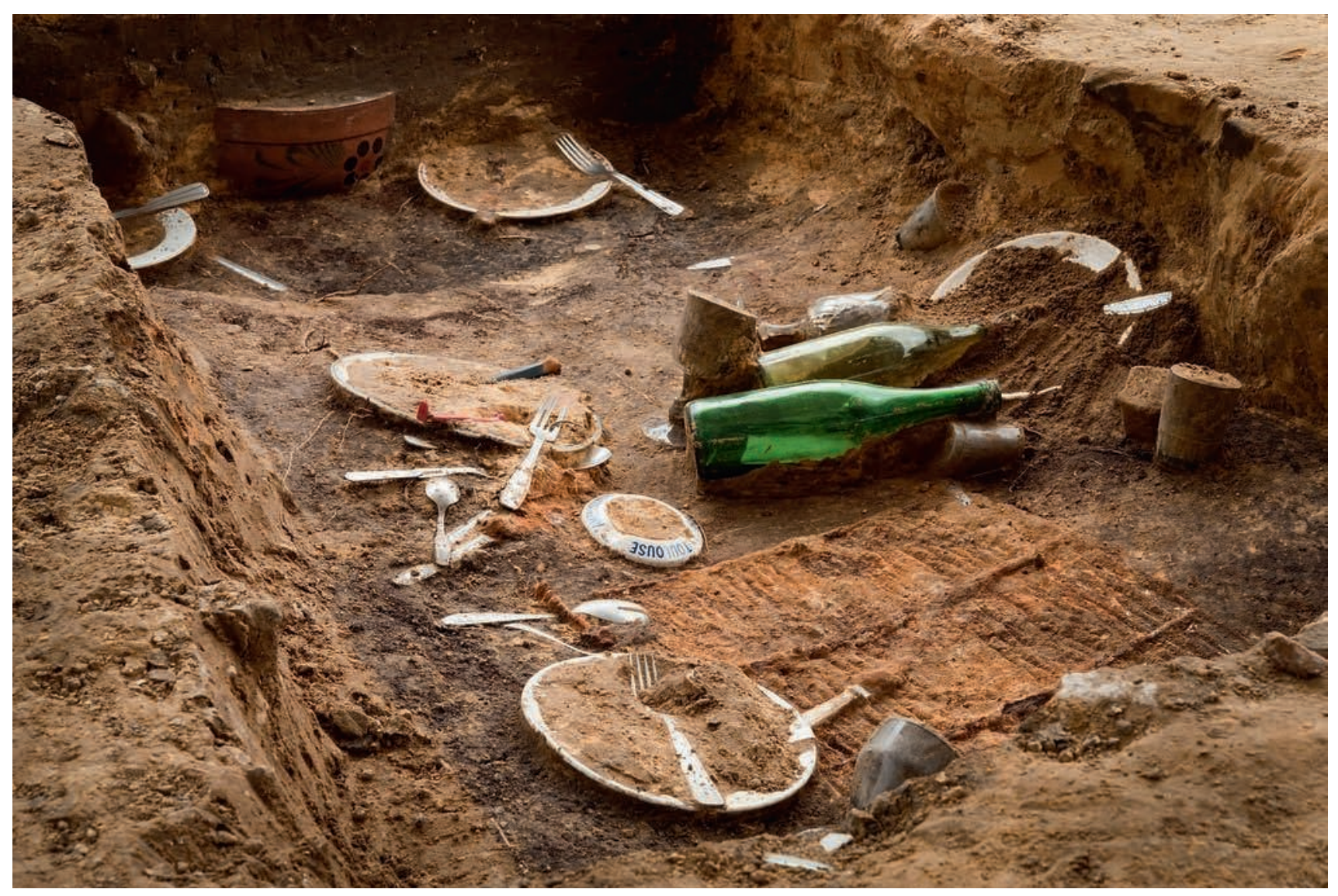


permettant de déterminer si ce phénomène représente une dominante. D'autre part, l'essor des stratégies performatives dans l'art européen et nord-américain depuis le milieu des années 1950 a contribué à mettre en avant des méthodes (partition, protocole, scénario, statement, script...) autorisant certains artistes à passer d'un régime sémiotique autographique (par exemple la peinture) à un régime allographique (par exemple la musique) de l'art, pour reprendre la terminologie de Nelson Goodman (Goodman 2005: 146-157). Le succès des procédés de délégation de production et la montée en puissance du conceptualisme et du post-conceptualisme dans l'art, de 1968 à nos jours, ont permis au protocole, à la partition, etc., de se muer en une forme de contrat social suffisamment routinisé pour devenir un cadre de médiation, de traduction et de transvaluation grâce auquel artistes, chargés de conservation et autres acteurs du monde de l'art font passer du vivant dans l'objet, voire l'y traduisent. Bien qu'une commune étymologie latine (curare, cura) fasse volontiers passer le conservateur (curator) des musées anglo-saxons pour quelqu'un prenant soin (le fameux care) de sa collection, reste à savoir si les conservateurs et assimilés peuvent être des soigneurs de micro-organismes, d'insectes, d'animaux, voire d'individus humains... II semble qu'ils ne le sont, le cas échéant, que de manière marginale, principalement durant le temps d'exposition et en s'assurant pour cela des collaborations diverses qui permettent à ces œuvres mixtes d'exister. Ils jouent parfois les négociateurs dans les polémiques que certaines œuvres, mettant à l'épreuve leur cadre de vie patrimonial, ne manquent pas de susciter. Les chargés de collection demeurent donc avant tout des soigneurs d'objets, c'est-à-dire des conservateurs, car ces œuvres mixtes y sont conservées sous ce strict format ontologique. Les petits arrangements ontologiques dont il a été question dans cet article se caractérisent par leur équilibre éminemment provisoire au sein d'un cadre objectuel qui, lui, paraît d'une solidité à toute épreuve.

Reste à savoir ce que ces dispositifs mixtes font à l'histoire de l'art: ils peuvent être l'occasion (renouvelée) de déconstruire les catégories ontologiques qui trament le champ de l'art, au premier rang desquelles celle d'objet, et de repenser à nouveaux frais une histoire sociale de l'art associée à l'anthropologie, qui intégrerait à ses enjeux d'enquête la question des collectifs d'humains et de non-humains.

Haute École des arts du Rhin cyrille.bret@hear.fr

\section{ci-contre}

fig. 8

Fouille archéologique du Déjeuner sous l'herbe de Daniel Spoerri à Jouyen-Josas. Enfoui depuis 1983, le banquet de Daniel Spoerri s'est décomposé, jusqu'à n'être qu'un souvenir. Pour en étudier les vestiges, vingt-sept ans plus tard, les premières fouilles archéologiques de l'histoire de l'art contemporain ont été organisées du 31 mai au 10 juin 2010. Photo (C) Inrap, Dist. RMN-Grand Palais / Denis Gliksman. ADAGP, Paris, 2016.
PS: Aux dernières nouvelles, le chat extrait du Chaquarium coule des jours paisibles et partage la vie d'une ex-collaboratrice de I'Institut d'art contemporain de Villeurbanne. Exposer de nouveau l'installation nécessiterait donc, soit de négocier avec cette personne le "prêt » de son animal (auquel elle tient beaucoup), soit de trouver (louer les services ou acheter) un nouveau chat auprès d'un éleveur, de la SPA ou d'une animalerie, d'engager un suivi vétérinaire et de respecter les lois relatives aux animaux en vigueur dans le pays d'exposition. 
Bibliographie

\section{Baxandall, Michael}

1985 L'CEil du Quattrocento.

Paris, Gallimard.

\section{Becker, Howard S.}

1988 Les Mondes de l'art. Paris, Flammarion.

\section{Bret, Cyrille}

2014 "Abolir la contradiction. Sciences physiques, philosophies extrême-orientales et stratégies plasticognitives dans les œuvres de George Brecht et Robert Filliou », in Bernard Guy (dir.), ASLC 2013. Troisièmes ateliers sur la contradiction. Paris,

Presses des Mines: 189-201.

\section{Calonje, Teresa (éd.)}

2014 Live Forever: Collecting Live Art. Cologne, Koenig Books.

\section{Cometti, Jean-Pierre}

2012 Art et facteurs d'art: ontologies friables. Rennes, Presses universitaires de Rennes.

\section{Descola, Philippe}

2005 Par-delà nature et culture. Paris, Gallimard.

\section{Domingez Rubio, Fernando}

2014 "Preserving the unpreservable: Docile and unruly objetcs at MoMA", Theory and Society 6(43) : 617-645.

Les collections d'art contemporain à l'épreuve du vivant à travers quelques cas remarquables. Par Cyrille Bret

\section{Filliou, Robert}

1998 Enseigner et apprendre, arts vivants. Paris et Bruxelles, Lebeer Hossmann.

\section{Francastel, Pierre}

2006 Études de sociologie de l'art. Paris, Tel-Gallimard.

\section{Goodman, Nelson}

2005 Langages de l'art.

Paris, Hachette-Pluriel.

\section{Latour, Bruno}

2006 Changer de société.

Refaire de la sociologie.

Paris, La Découverte.

\section{Moulin, Raymonde}

2003 Le Marché de l'art.

Mondialisation et nouvelles technologies. Paris, Flammarion.

\section{O'Doherty, Brian}

2008 White Cube. L'espace de la galerie et son idéologie. Zurich et Villeurbanne, JRP/Ringier (" Lectures Maison rouge»).

\section{Poinsot, Jean-Marc}

1999 Quand l'œuvre a lieu.

L'art exposé et ses récits autorisés.

Genève et Villeurbanne,

MAMCO-Art Édition.

\section{Pommier, Édouard}

2007 Comment l'art devint l'Art

dans I'Italie de la Renaissance.

Paris, Gallimard.

\section{Quenault, Samuel}

2013 "CEuvres composites/œuvres vivantes", CeROArt [en ligne], disponible sur: https://ceroart. revues.org/3450 (consulté le 8 novembre 2015).

\section{Yaneva, Albena}

2003 «When a bus met a museum: installation ", Museum and Society $1(3)$ : 116-131. page 146 et ci-contre Fouille archéologique du Déjeuner sous I'herbe de Daniel Spoerri à Jouy-en-Josas. Enfoui depuis 1983, le banquet de Daniel Spoerri s'est décomposé, jusqu'à n'être qu'un souvenir. Pour en étudier les vestiges, vingt-sept ans plus tard, les premières fouilles archéologiques de l'histoire de l'art contemporain ont été organisées du 31 mai au 10 juin 2010. Photo @ Inrap, Dist.

RMN-Grand Palais / Denis Gliksman. ADAGP, Paris, 2016. 
